\title{
Electroconvulsive Shock- or Puromy cin-Induced Retention Deficits in Goldfish Given Two Active-Avoidance Sessions ${ }^{1}$
}

\author{
ALAN D. SPRINGER and BERNARD W. AGRANOFF \\ Neuroscience Laboratory, University of Michigan, Ann Arbor, Michigan 48109
}

In a factorial design, goldfish received 15 active-avoidance training trials on Days 1, 7, and 13 followed by electroconvulsive shock (ECS) or no treatment after the training session on Days 1 and 7. The final retention deficits observed when ECS was given after the first session only or after the second session only were similar. Fish given ECS after both sessions showed a cumulative deficit that approximated the sum of the two effects. Similar results were obtained with puromycin. These data support the hypothesis that ECS and puromycin impair only recent learning. Retrograde amnesia gradients confirmed that the efficacy of these amnestic agents was not altered by a second administration.

Intracranial injection of inhibitors of protein or of RNA synthesis to goldfish before or just following a training session disrupts fixation of long-term memory of the training session (Agranoff, 1974). Electroconvulsive shock (ECS) is also effective in producing amnesia (Davis et al., 1965), but the relationship of its mechanism of action to that of the antibiotics is unknown. Recent experiments with goldfish have indicated that ECS, like the protein and RNA synthesis inhibitors, can exert its effect proactively in a multitrial active-avoidance task (Springer et al., 1975). Since acquisition is demonstrated to be unimpaired, this result suggests that the amnestic effect of ECS is related to a sequela of the treatment rather than to events occurring at the time of shock administration, such as the resulting convulsions. A puzzling aspect of ECS effects in rats is the claim that memory can become refractory to disruption by repeated treatments (Nachman and Meinecke, 1969). If goldfish memory also becomes refractory to ECS the finding might well provide additional insights into the nature of ECS action. It was therefore of interest to investigate ECS impaired retention in goldfish following a second administration. The possible loss of effectiveness of a second puromycin injection after a second training session was also examined.

1This research was supported by Grants NIMH 2R01MH12506 and NSF BMS75-03810. 


\section{EXPERIMENT 1}

\section{Method}

Subjects. One hundred fifty-nine common goldfish (Carassius auratus), weighing 8-11 g and 6-7 $\mathrm{cm}$ in length from snout to caudal peduncle, were obtained from Ozark Fisheries, Stoutland, Missouri. The fish were housed in 750-liter tanks for approximately 1-2 weeks and were then placed in separate 1.5 -liter tanks for 1-2 days prior to Day 1 of the experiment. They were maintained at $20 \pm 1{ }^{\circ} \mathrm{C}$ in continuous light and were not fed. All studies were performed between January and March.

Apparatus and procedure. Active-avoidance training was performed in an aquatic shuttlebox which differed from a previously described box (Agranoff, 1971) in that it contained a 6-mm-thick, black Plexiglas partition that completely divided the box into two compartments. A $4 \times 3 \mathrm{~cm}$ hole was centered in the barrier dividing the two compartments. The bottom of the hole was $3 \mathrm{~cm}$ from the floor of the tank and the water level in the tank was $5 \mathrm{~cm}$.

On Day 1, fish were acclimated in individual shuttleboxes in the dark for 5 min prior to the onset of 15 training trials. Each trial lasted $1 \mathrm{~min}$ and began with $15 \mathrm{sec}$ of light presented on the side of the box occupied by the fish, followed by $20 \mathrm{sec}$ of light paired with shock $(3.5 \mathrm{~V}, 60 \mathrm{~Hz}, \mathrm{rms}$, 100 -msec duration, 2.5-sec interpulse interval). An escape response was recorded when a fish crossed the barrier in response to shock and an avoidance response was recorded when a fish crossed the barrier prior to shock onset. Trials were initiated every $60 \mathrm{sec}$, so that escape or avoidance responses terminated the trial (light and shock off) and initiated an intertrial interval of at least $25 \mathrm{sec}$ of darkness. A failure to escape was recorded when a fish failed to cross into the safe compartment within the first $35 \mathrm{sec}$ of a trial.

The subjects were divided into four groups. Two groups (Groups 1 and 2) received retro-orbital ECS $(0.1 \mathrm{sec}, 30 \mathrm{~mA}, 60 \mathrm{~Hz}, \mathrm{rms})$ (Springer et al., 1975) upon the termination of Day 1 training. The two remaining groups (Groups 3 and 4) did not receive ECS. Following 15 training trials on Day 7, Groups 1 and 4 received ECS. Fifteen additional training trials were given to all groups on Day 13.

\section{Results}

A multiple regression analysis was performed using the number of avoidances $(A)$, the total number of shocks $(S)$, and the failures to escape $(F)$ on Day 1. This analysis used the data of the 70 fish that did not receive ECS on Day 1 (Group 4) and resulted in an equation that predicted the number of avoidances that should occur on Day 7, based on their performance on Days 1 
and $7[P=1.30+2.89 \log (A+1)+0.02 S]$. This equation was then applied to all fish in the experiment to predict Day 7 avoidances. Similarly, another equation was derived from Group 4 that was used to predict Day 13 avoidances from Day 1 scores $[P=3.74+3.04 \log (A+1)-0.62 F+.07 S]$.

Analysis of the difference between achieved (A) and predicted (P) avoidances (A-P) for Day 7 using dependent $t$ tests showed a significant retention deficit in the two groups that received ECS immediately after training on Day 1 (Groups 1 and 2, Table 1). ECS after training on Day 7, in fish that also received ECS on Day 1, effectively impaired retention for Day 7 acquisition as tested on Day 13 (Group 1). ECS given to fish on Day 7 but not Day 1 (Group 3) similarly resulted in a significant retention deficit. ECS given on Day 1 but not on Day 7 also resulted in a significant retention deficit on Day 13 (Group 2).

Two-tailed independent $t$ tests were used to compare the Day 7 mean A-P scores for the four groups. The two groups receiving ECS on Day 1 (Groups 1 and 2) did not differ $(P>0.5)$, nor did the two groups not receiving ECS (Groups 3 and 4), $(P>0.5)$. Both Groups 1 and 2 differed significantly from Groups 3 and $4(P<0.01)$.

Analysis of Day 13 A vs. P scores with dependent $t$ tests found significant retention deficits in Groups 1,2 , and 3 . A $2 \times 2$ unequal $n$ ANOVA on the A-P retention socres for Day 13 found a significant effect of ECS given on Day $1[F(1,155)=14.03, P<0.001]$, as well as a significant effect of ECS given on Day $7[F(1,155)=39.51, P<0.001]$. The interaction of ECS on Day 1 and ECS on Day 7 did not achieve significance $(P>0.25)$, suggesting that the effects of the two ECS treatments were additive. The additive effects of the two treatments are apparent in Fig. 1.

\section{Discussion}

The deficit in Group 1 relative to Group 4 increased from Day 7 to Day 13 , suggesting that a second ECS can impair memory. Furthermore, examination of avoidances from Day 1 to Day 7 and Day 13 in Group 1 reveals no significant increase (2.00-2.31-2.83), suggesting that the two ECS treatments have impaired retention of both Day 1 and Day 7 acquisition. In contrast, the deficit in Group 2 is not increased from Day 7 to Day 13, indicating that following the first training-ECS session, acquisition and retention is not further impaired. Group 3 indicates that a training-ECS session that follows a prior training-no ECS session results in a deficit for the second session.

\section{EXPERIMENT 2}

Although the data of Experiment 1 indicate that two successive training-ECS sessions do not result in a progressive loss of amnestic action, it 


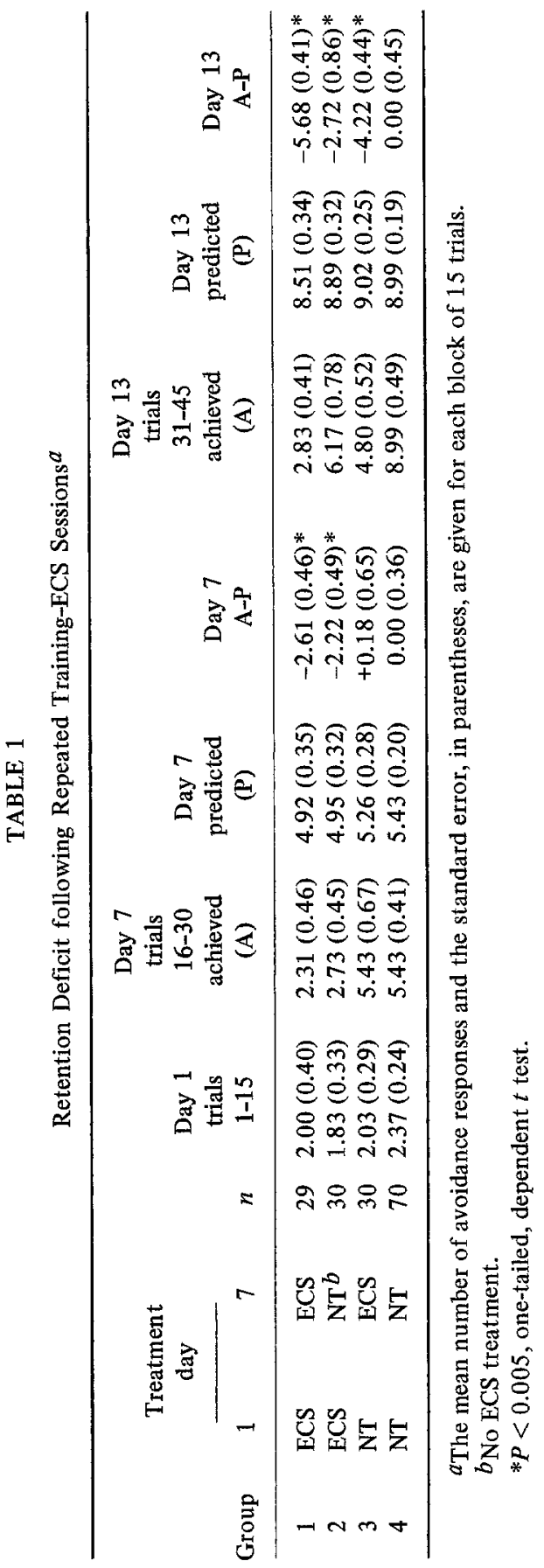




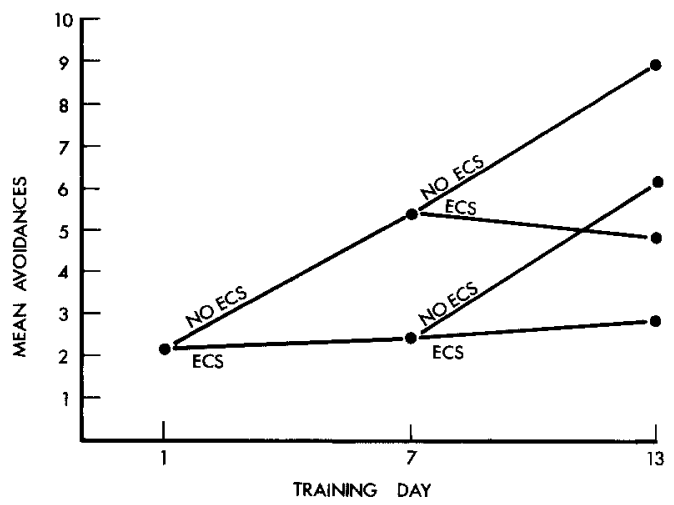

Fig. 1. Avoidances of groups that received ECS or no ECS following each training session. For purposes of clarity, several data points for Days 1 and 7 were averaged. See Table 1 for complete details.

still remains possible that a different, possibly more sensitive test might reveal a trend in that direction. Graded intensity of ECS alters the rate of development of retrograde amnesia (RA) following ECS in rats (Gold et al., 1973). Since the slope of the consolidation curve can thus reflect the efficacy of ECS, we compared the rate of development of insusceptibility to ECS for the first training session with that obtained in groups that received immediate or delayed ECS after the first training session and various delays following the second training session. If a second training-ECS session results in less amnesia, the first and second gradients should converge, i.e., the second gradient should be steeper than the initial gradient. Alternatively, if ECS following the second session is as effective in inducing amnesia as ECS following the first session, the two gradients should be parallel.

\section{Method}

Four hundred twenty-seven fish similar to those described in the initial experiment were used. Apparatus, shock, and ECS parameters were those described. Three groups of fish were trained on Day 1 and given ECS either 0, 5 , or $24 \mathrm{hr}$ following training; a fourth group did not receive ECS. All fish were given 15 additional trials on Day 7 . The group that did not receive ECS on Day 1 was divided into four groups which received either no ECS or ECS 0,5 , or $24 \mathrm{hr}$ after training on Day 7 (Groups 1, 2, 3, and 4). Fish that received ECS $24 \mathrm{hr}$ after training on Day 1 were divided into three groups that received either no ECS, ECS delayed by $24 \mathrm{hr}$, or ECS immediately after training on Day 7 (Groups 5, 6, and 7). Animals that received ECS $5 \mathrm{hr}$ after training on Day 1 were divided into two groups and received either immediate 
or no ECS following training on Day 7 (Groups 8 and 9). Fish that received ECS immediately after training on Day 1 were divided into four groups that received either no ECS, 0-hr delay ECS, 5-hr delay ECS, or 24-hr delay ECS after training on Day 7 (Groups 10, 11, 12, and 13). All fish received 15 additional training trials on Day 13. The four groups reported in Experiment 1 were run at the same time as the groups reported in the present experiment and are included in Experiment 2.

\section{Results}

The multiple regression analysis described in Experiment 1 was used to predict Day 7 and Day 13 avoidances. No ECS or ECS administered 0, 5, or $24 \mathrm{hr}$ after training on Day 1 resulted in a gradient on Day 7 (see Table 2 and Fig. 2). When ECS was readministered after various delays following training on Day 7 to the groups that had received no ECS or ECS 0,5 , or $24 \mathrm{hr}$ after training on Day 1, four gradients were obtained on Day 13. While the exact details of the retrograde amnesia gradient have not been established with the present apparatus (hole-barrier) and parameters, comparison of the four Day 13 gradients with the Day 7 gradient (Fig. 2) suggests that the second ECS

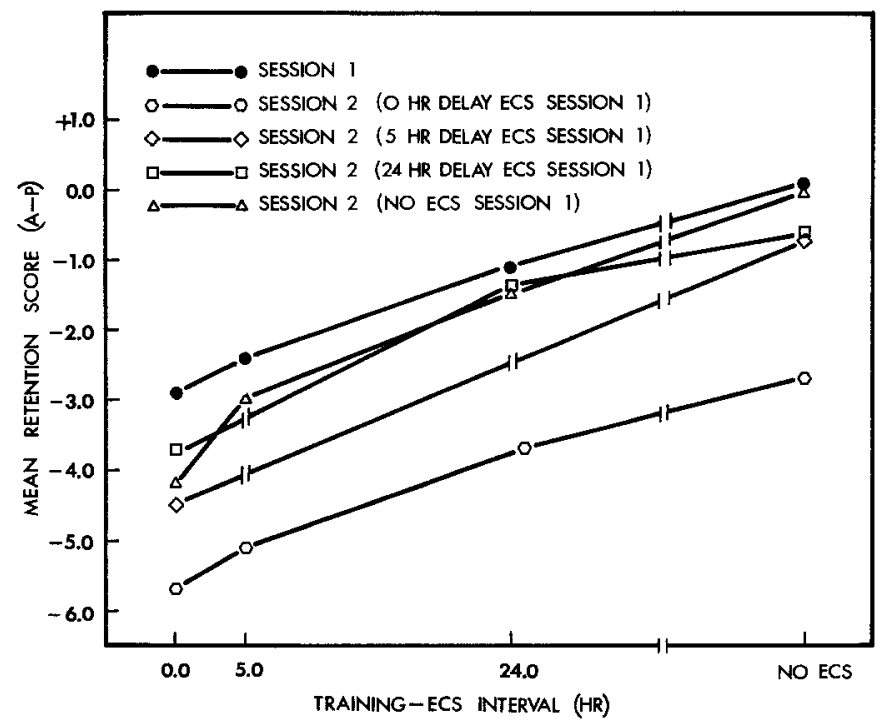

Fig. 2. Retention deficits when ECS is administered at various delays following the first or both training sessions. Lines are drawn between points to demonstrate similarities in slopes and do not indicate the precise shape of the retrograde amnesia gradient (see text). Session 1 refers to deficits for Day 1 training as measured on Day 7 (see Day 7 A-P, Table 2). Session 2 refers to deficits for Day 7 training as measured on Day 13 (see Day 13 A-P, Table 2). Abscissa: training-ECS delay for either Day 1 or Day 7 training. 


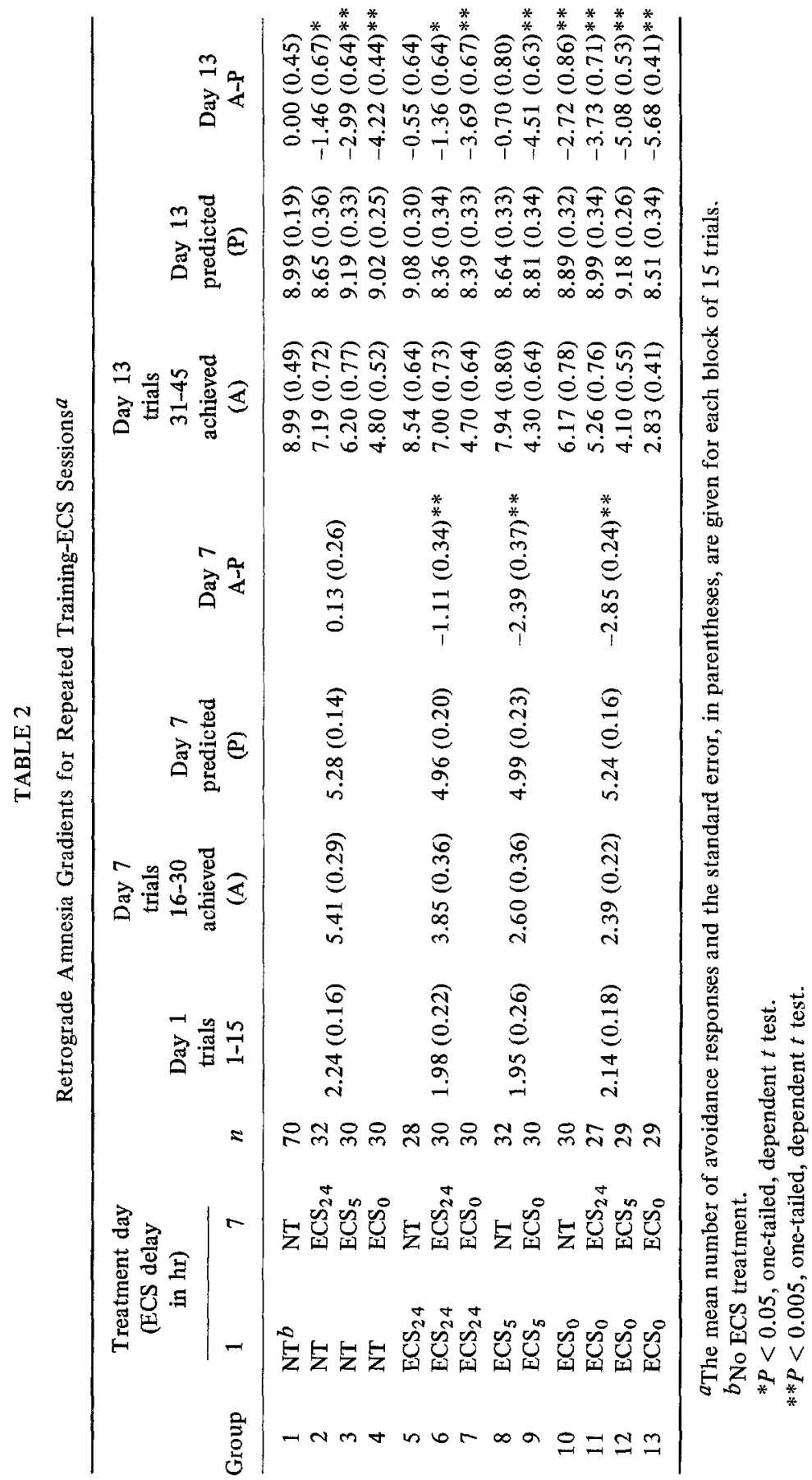


treatment was as effective in producing a retention deficit as the first ECS since the gradients were similar.

Correlated $t$ tests between achieved and predicted avoidances (A vs. P) for Day 7 indicated significant retention deficits in the groups receiving ECS 0,5 , or $24 \mathrm{hr}$ following training (Table 2). An ANOVA on the mean A-P scores for Day 7 proved significant $[F(3,423)=24.81, P<0.001]$ and two-tailed, independent $t$ tests were used to determine the source of the effect. The groups that did not receive ECS following training differed significantly from all three groups that received ECS following training $(P<0.01)$, while the 0 - and 5-hr ECS delay groups did not differ from one another but did differ from the 24-hr ECS delay group $\left(P_{\mathrm{S}}<0.02\right)$.

Dependent, one-tailed $t$ tests between $\mathrm{A}$ and $\mathrm{P}$ scores for Day 13 (Table 2) found significant retention deficits in Groups $2,3,4,6,7,9,10,11,12$, and 13. As the data for Day 13 do not represent a complete factorial design, the four gradients (Fig. 2) were analyzed separately (i.e., Groups 1-4, 5-7, 8-9, and 10-13). A one-way ANOVA across Groups 1-4 proved significant $[F(3,158)=12.01, P<0.001]$. Two-tailed, independent $t$ tests between Groups $1-4$ found that Group 1 differed from Groups 3 and $4(P<0.001)$ and Group 2 differed from Group $4(P<0.01)$. An ANOVA across Groups 5-7 achieved significance $[F(2,85)=6.22, P<0.005]$. Two-tailed, independent $t$ tests between Groups 5-7 found that Groups 5 and 6 differed from Group $7 \quad(P<0.02)$. A $t$ test comparing the means of Groups $10-13$ also achieved significance $(P<0.001)$. A $t$ test comparing the means of Groups 8 and 9 achieved significance $(P<0.001)$. An ANOVA across Groups $10-13$ also achieved significance $[F(3,111)=4.24, P<0.025]$, and two-tailed, independent $t$ tests found that Group 10 differed from Groups 12 and $13(P<0.05)$ and Group 11 differed from Group $13(P<0.05)$.

\section{Discussion}

A gradient was obtained on Day 7 in groups of fish that received 0-, 5-, or 24-hr delay ECS or no ECS (NT) following training on Day 1 (Fig. 2). Gradients were also obtained on Day 13 in groups of fish that had previously received either 0-, 5-, or 24-hr delay ECS or no ECS on Day 1 and various ECS delays following training on Day 7. Examination of Fig. 2 reveals that the gradients obtained for the second training session (Day 7) do not intersect or converge with the gradient for Day 1 training. This is particularly evident in comparing the slope of the gradient of fish that received immediate ECS after training on Day 1 and various ECS delays following training on Day 7 with the Day 1 gradient. These data further indicate that the efficacy of ECS in inducing a retention deficit is undiminished when ECS is given following two successive training sessions. Moreover, ECS is still effective in inducing 
amnesia when administered following a second training session even when its delayed administration after the first training session had little effect.

It is unlikely that the deficits seen on Day 13 in Groups 6, 7, 9, 11, 12, and 13 are a consequence of sickness from having received two ECS treatments. If two ECS treatments were debilitating to the fish, the retention deficits on Day 13 should be equivalent in Groups 6,7,9,11,12, and 13, all of which received two ECS treatments. However, as the retention deficits on Day 13 in Groups 6 and 13 are significantly different $(P<0.001)$, it is unlikely that two ECS treatments produce sickness. The deficit on Day 7 in the 24-hr delay group (Groups 5,6, and 7) suggests an RA gradient that is longer than that observed in a somewhat different paradigm (Davis et al., 1965) but is consistent with recent findings (Springer et al., 1975). In addition, long gradients have been observed in other species (Cherkin, 1969; Robustelli et al., 1969).

Somewhat puzzling are the deficits seen in Groups 2 and 5 (NT + $\mathrm{ECS}_{24}$ and $\left.\mathrm{ECS}_{24}+\mathrm{NT}\right), 3$ and $8\left(\mathrm{NT}+\mathrm{ECS}_{5}\right.$ and $\left.\mathrm{ECS}_{5}+\mathrm{NT}\right)$, and 4 and $10\left(\mathrm{NT}+\mathrm{ECS}_{0}\right.$ and $\left.\mathrm{ECS}_{0}+\mathrm{NT}\right)$. These pairs of groups do not differ from one another in overall combined treatments, but rather in the temporal relation between treatments. Nevertheless, the groups that received the NT + ECS treatment evidenced a larger deficit on Day 13 than their respective counterpart group which received the ECS + NT treatment. An independent two-tailed $t$-test between the pooled data of Groups 2, 3, and 4 (NT $+\mathrm{ECS}_{24}$; $\left.\mathrm{NT}+\mathrm{ECS}_{5} ; \mathrm{NT}+\mathrm{ECS}_{0}\right)$ vs. 5,8 , and $10\left(\mathrm{ECS}_{24}+\mathrm{NT} ; \mathrm{ECS}_{5}+\mathrm{NT} ; \mathrm{ECS}_{0}+\right.$ NT) achieved significance $(P<0.02)$. This suggests that no ECS after training on Day 1 followed by ECS after training on Day 7 (NT + ECS) does not result in an equivalent deficit on Day 13 as does ECS after training on Day 1 followed by no ECS after training on Day 7 (ECS + NT).

There is a number of possible explanations that may account for the difference. The most parsimonious is based on forgetting; acquisition on Day 1 is not equivalent to acquisition on Day 7 as far as influencing performance on Day 13. Since the interval between Day 1 acquisition and Day 13 is twice as long as that of Day 7 and Day 13, it is possible that Day 1 acquisition is only partially retained by Day 13 while acquisition of Day 7 is completely retained. If, in fact, Day 7 acquisition is weighted more heavily than Day 1 acquisition, ECS on Day 1 may have a lesser effect on Day 13 performance than ECS on Day 7. To test this possibility, two groups of fish received 15 trials on Day 1 and were retrained on Day 7 or Day 13. The group trained on Day 1 and retrained on Day $7(n=29)$ evidenced 1.73 more avoidances on Day 13 than a group trained on Day 1 and retrained on Day $13(n=35)$. Thus, the difference between the ECS + NT and NT + ECS groups can be explained in terms of forgetting in the NT + ECS condition, thus obviating reminder or reinstatement interpretations. 


\section{EXPERIMENT 3}

Inasmuch as ECS retained its potential to disrupt memory over repeated administration, it was of interest to determine whether these findings also generalize to, puromycin-induced amnesia. The present study consisted of a 3 $\times 3$ factorial design with animals receiving either no puromycin or puromycin immediately or $24 \mathrm{hr}$ following training on Day 1 and Day 7.

\section{Method}

Three hundred twenty-two fish were trained using the procedures described in Experiment 1. Three groups (Groups 1-3) did not receive any treatment following Day 1 training; three groups (Groups 4-6) received an intracranial injection of puromycin $(130 \mu \mathrm{g} / 10 \mu \mathrm{l}$ of saline) $24 \mathrm{hr}$ following Day 1 training; three groups (Groups 7-9) were injected with puromycin immediately following Day 1 training. All fish were given 15 training trials on Day 7. Groups 1, 2, and 3 received either no injection, a 24-hr delay, or immediate injection of puromycin following Day 7 training; Groups 4-6 and 7.9 received similar treatments (see Table 3 for details). On Day 13 all fish received 15 additional training trials.

Results

A multiple regression analysis (see Experiment 1), based on Day 1 performance of Group 1, was used to predict Day 7 and Day 13 avoidances. Groups of fish received either no puromycin, immediate puromycin, or 24-hr delayed puromycin following Day 1 training and a gradient was obtained on Day 7 (see Table 3 and Fig. 3). Each of the three groups received either no puromycin or puromycin 0 or $24 \mathrm{hr}$ following Day 7 training, and retraining on Day 13 resulted in similar gradients. As with ECS, puromycin also effectively impairs retention following repeated training-puromycin sessions. It should be noted that these curves reflect only the limits of the RA gradient and do not describe the actual shape of the RA gradient.

Dependent $t$ tests were used to determine the significance of the deficit (A vs. P) on Day 7 (Table 3). Only the group receiving puromycin immediately following Day 1 training evidenced a significant deficit on Day 7. Similar analysis of Day 13 A vs. $P$ scores found significant deficits in Groups $3,6,7,8$, and 9 .

A one-way ANOVA comparing the Day 7 A-P scores achieved significance $[F(2,319)=11.92, P<0.001]$. Two-tailed, independent $t$ tests found that fish receiving either no injection or a 24 -hr delayed injection following Day 1 training did not differ from one another but both differed from the group that received puromycin immediately after Day 1 training $\left(P_{\mathrm{S}}<0.001\right)$. 


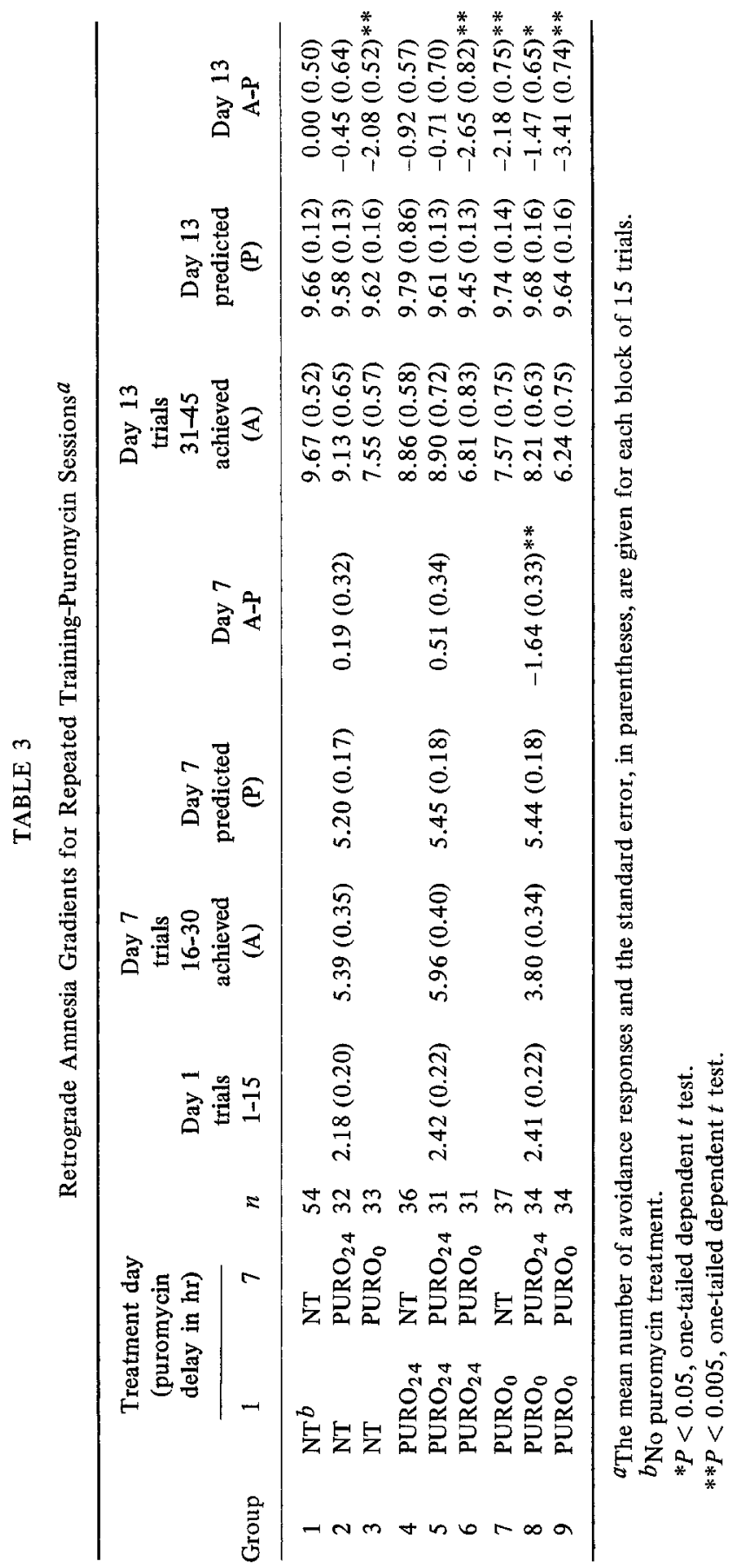




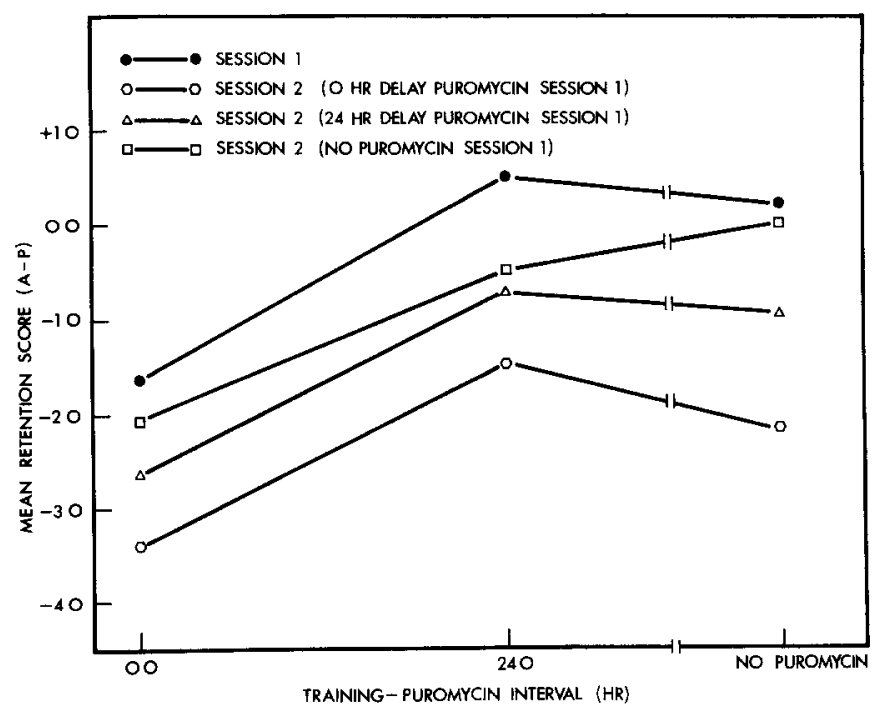

Fig. 3. Retention deficits when puromycin is administered at various delays following the first or both training sessions. Lines are drawn between points to demonstrate similarities in slopes. These points indicate limits and do not indicate the precise shape of the retrograde amnesia gradient. Session 1 refers to deficits for Day 1 training as measured on Day 7 (see Day 7 A-P, Table 3). Session 2 refers to deficits for Day 7 training as measured on Day 13 (see Day 13 A-P, Table 3). Abscissa: training-puromycin delay for either Day 1 or Day 7 training.

Day 13 A-P scores were analyzed with a $3 \times 3$ ANOVA. Both main effects (puromycin on Day 1 and puromycin on Day 7) achieved significance $[F(2,313)=4.61, P<0.025] ;[F(2,313)=7.09, P<0.005]$. The interaction was not significant $(P>0.50)$, indicating that the effects of each puromycin are additive. Independent, two-tailed $t$ tests were used to determine the source of the Day 1 puromycin effect (Group $1+2+3$ vs. $4+5+6$ vs. $7+8+9$ ). The no-puromycin treatment did not differ from the 24 -hr delay puromycin treatment $(P>0.10)$ but both differed from the immediate puromycin treatment $\left(P_{s}<0.01\right)$. Similar tests were used to determine the source of the Day 7 puromycin effect (Groups $1+4+7$ vs. $2+5+8$ vs. $3+6+9$ ). These tests revealed that the no-injection and 24-hr delay puromycin injection conditions did not differ $(P>0.50)$, while both these treatment groups differed from the immediate puromycin treatment $(P s<0.01)$.

\section{Discussion}

The results of the present experiment are consistent with those of Experiments 1 and 2. A gradient for session 1 was obtained with puromycin 
(Fig. 3) since the immediate and 24-hr delay puromycin groups differed significantly. Similarly, three gradients were obtained for session 2 (Fig. 3), and a comparison of the initial gradient with the latter three gradients found that the RA gradients for session 2 do not converge upon the RA gradient for session 1, i.e., they are not steeper than the gradient for session 1 . Consequently, it appears that the efficacy of puromycin to induce a retention deficit is not attenuated over two successive training-puromycin sessions. Furthermore, it is unlikely that two injections of puromycin are inducing sickness on Day 13, since Group 5, which received two 24-hr delay puromycin injections, does not show a significant retention deficit on Day 13.

\section{GENERAL DISCUSSION}

Although blockers of protein synthesis and ECS are both amnestic in the goldfish, there are differences as well as similarities in their mechanisms of action. While ECS produces some decrement in brain protein synthesis, the amount of inhibition is insufficient to produce amnesia, based on protein inhibitor studies (Agranoff, 1965; Dunn et al., 1974). ECS, like the protein and RNA synthesis inhibitors, can produce a proactive effect, although with ECS the duration of the effect appears to be quite brief. With both the inhibitors of macromolecular synthesis and ECS, retraining shortly after a treatment reveals that memory is still present, even though it will be undetectable within a few days (Springer et al., 1975). In the case of ECS, the subsequent memory loss occurs more rapidly than in the case of the inhibitors. In the present study, the two classes of amnestic agents exhibit another similarity: Both demonstrate an additive effect when given after each of two training sessions.

The repeated training-amnestic agent approach used in the present studies incorporates aspects of several effects encountered in investigations of memory mechanisms, including reinstatement, familiarization, and reminder. In the resinstatement paradigm, subjects are trained and are exposed at some later time to training stimuli, followed by an amnestic agent treatment (Misanin et al., 1968), given at a time when it can no longer produce amnesia. Exposure to training stimuli is thought to reinstate the original memory, thus permitting ECS to impair retention of training. Accordingly, in Group 3 (NT + ECS; Table 1), session 2 should activate memory of session 1, and ECS following session 2 should impair memory of both sessions. The deficit on Day 13 in Group 3 should thus be as great at that of Group 1 (ECS + ECS) which received ECS after each session. Contrary to the prediction of a reinstatement model, the deficit in Group 3 is significantly less than that observed in Group 1.

In a familiarization paradigm (Miller, 1970), subjects are exposed to 
training stimuli and subsequently given training and an amnestic agent treatment. This model, in contradistinction to the reinstatement model, predicts that preexposure to training stimuli should decrease the observed memory loss. The familiarization model is evident in Group 3 (NT + ECS; Table 1) since a session without ECS is followed by a session with ECS. Therefore, ECS should not impair retention of either session and on Day 13, Group 3 should be equivalent to Group 4 (NT + NT). However, a significant difference is observed in Group 3 as compared to Group 4 (Table 1). Furthermore, measurable amnesia was observed even when ECS was delayed after the second session (Table 2, Groups 1-4).

The reminder paradigm is provided by Group 2 (ECS + NT; Table 1). Exposure to training stimuli subsequent to training and amnestic agent treatment should attenuate the observed amnesia (Miller and Springer, 1973). Thus, on Day 13, Group 2 should not show a deficit relative to Group 4 (NT $+\mathrm{NT}$ ). There is no evidence to support a reminder effect in fish given ECS immediately following training since a significant deficit is observed in Group 2 as compared to Group 4 (Table 1). A reminder effect is evident when ECS is delayed following training (Table 2; Groups 5 and 8 ) since these groups do not evidence a retention deficit relative to the group that did not receive ECS after each session (Group 1). The result suggests that memory can be recovered in instances where an amnestic agent does not fully block fixation. This observation is consistent with findings in other species (Cherkin, 1972; Gold et al., 1973).

In previous studies which examined these effects, the cues used to reinstate, familiarize, or remind are of necessity elements of the training trial, but are not a complete trial. The reason for this is that when a one-trial passive-avoidance paradigm is used, it results in maximal learning in a single trial and therefore a training trial cannot serve as a reminder cue. Since multiple training trials were used in the present studies and asymptotic learning was not achieved in a single session, it was possible to use a session as a reminder. Since the session would seem at least as valid or perhaps a more valid cue than its elements (e.g., punishing shock), it is of interest that under these conditions the present experiments do not confirm the predictions of the reinstatement, familiarization, and reminder models.

Davis and Hirtzel (1970) have reported that a reinstatement effect for memory of shock-avoidance learning in goldfish is time dependent over a period of 4-6 hr. Therefore, it is possible that a reinstatement, reminder, or familiarization effect might have been observed in the present study had the interval between the training sessions been reduced from 7 days. Similarly, the failure to observe a memory deficit in rats following a second training-ECS or training- $\mathrm{CO}_{2}$ treatment (Nachman and Meinecke, 1969) or second traininghypothermia treatment (Riccio and Stikes, 1969) may be due to the use of a 1-day interval between each session. While differences in species and paradigm 
preclude rigorous comparisons of these studies with the present one, it should be noted that the use of partial learning and multiple trials provides learning curves and avoids ceiling effects inherent in the one-trial passive-avoidance paradigm. For these reasons, the present approach is particularly useful in determining additive effects of multiple training-amnestic agent sessions and putative interactive processes including reinstatement, reminder, and familiarization effects.

In the present studies, it is apparent that both ECS and puromycin selectively affect recent training. When either amnestic agent is administered after session 1, it appears to affect retention of session 1 but not of session 2 , and when the agent is administered after session 2, it seems to impair retention of only that session. When given after each of two sessions, the combined effect is additive. Consequently, the present findings are viewed as further support of the hypothesis that amnestic agents disrupt the fixation of only recently acquired information.

\section{REFERENCES}

Agranoff, B. W. (1965). Molecules and memories. Perspect. Biol. Med. 9, 13-22.

Agranoff, B. W. (1971). Effects of antibiotics on long-term memory formation in the goldfish. In W. K. Honig and P. H. R. James (Eds.), "Animal Memory." New York: Academic Press.

Agranoff, B. W. (1974). Biochemical concomitants of the storage of behavioral information. In L. Jaenicke (Ed.), "Biochemistry of sensory functions," pp. 597-623. Berlin: Springer-Verlag.

Cherkin, A. (1969). Kinetics of memory consolidation: Role of amnesic treatment parameters. Proc. Natl. Acad. Sci. Wash. 63, 1094-1101.

Cherkin, A. (1972). Retrograde amnesia in the chick: Resistance to the reminder effect. Physiol. Behav. 8, 949-955.

Davis, R. E., Bright, P. J., and Agranoff, B. W. (1965). Effect of ECS and puromycin on memory in fish. J. Comp. Physiol. Psychol. 60, 162-166.

Davis, R. E., and Hirtzel, M. S. (1970). Environmental control of ECS-produced retrograde amnesia in goldfish. Physiol. Behav. 5, 1089-1092.

Dunn, A. D., Giuditta, A., Wilson, J. E., and Glassman, E. (1974). The effect of electroshock on brain RNA and protein synthesis and its possible relationship to behavioral effects. In M. Fink, S. Kety, J. McGaugh, and T. A. Williams (Eds.), "Psychobiology of Convulsive Therapy." Washington D. C.: V. H. Winston and Sons.

Gold, P. E., Haycock, J. W., and McGaugh, J. L. (1974). Retrograde amnesia and the "reminder effect." Science 186, 1135-1136.

Gold, P. E., Macri, J., and McGaugh, J. L. (1973). Retrograde amnesia gradients: Effects of direct cortical stimulation. Science 179, 1343-1345.

Miller, R. R. (1970). Effects of environmental complexity on amnesia induced by electroconvulsive shock in rats. J. Comp. Physiol. Psychol. 71, 267-275.

Miller, R. R., and Springer, A. D. (1973). Amnesia, consolidation, and retrieval. Psychol. Rev. 80, 69-79. 
Misanin, J. R., Miller, R. R., and Lewis, D. J. (1968). Retrograde amnesia produced by electroconvulsive shock after reactivation of a consolidated memory trace. Science 160, 554-555.

Nachman, M., and Meinecke, R. O. (1969). Lack of retrograde amnesia effects on repeated electroconvulsive shock and carbon dioxide treatments. J. Comp. Physiol. Psychol. 68, 631-636.

Riccio, D. C., and Stikes, E. R. (1969). Persistent but modifiable retrograde amnesia produced by hypothermia. Physiol. Behav. 4, 649-652.

Robustelli, F., Geller, A., and Jarvik, M. E. (1969). Temporal gradient of 23 hours with electroconvulsive shock and its implications. Commun. Behav. Biol. 4, 79-84.

Springer, A. D., Schoel, W. M., Klinger, P. D., and Agranoff, B. W. (1975). Anterograde and retrograde effects of electroconvulsive shock and of puromycin on memory formation in the goldfish. Behav. Biol. 13, 467-481. 